\title{
Futurité, origine des institutions économiques
}

Futurity, origin of the economic institutions

Jean-Jacques Gislain

\section{(2) OpenEdition}

Journals

Édition électronique

URL : http://journals.openedition.org/ei/5828

DOI : 10.4000/ei.5828

ISSN : 2553-1891

\section{Éditeur}

Association Économie et Institutions

\section{Référence électronique}

Jean-Jacques Gislain, «Futurité, origine des institutions économiques », Économie et institutions [En ligne], 25 | 2017, mis en ligne le 20 décembre 2017, consulté le 10 décembre 2020. URL : http:// journals.openedition.org/ei/5828; DOI : https://doi.org/10.4000/ei.5828

Ce document a été généré automatiquement le 10 décembre 2020.

Revue Économie et institutions 


\section{Futurité, origine des institutions économiques}

Futurity, origin of the economic institutions

Jean-Jacques Gislain

Je remercie Sylvie Morel, Bruno Théret et les évaluateurs de la Revue pour leurs remarques pertinentes.

La futurité est le principe le plus important de

l'économie.

(Commons 1934b, 125)

Le temps est totalement institutionnel.

(Commons 1934a, 642)

1 Les théories des institutions économiques se posent rarement la question de l'origine de ces institutions et la plupart les construisent sur la base de leur secondarisation par rapport à une instance première qu'il faudrait "réguler»: le Marché, le Mode de production capitaliste, le Système social. Seule l'approche institutionnaliste, en particulier celle de John Rogers Commons (1862-1945), conçoit l'activité économique comme d'abord instituée. La question de l'origine des institutions économiques est alors première dans la compréhension de l'activité économique. La réponse à cette question à partir du principe de futurité, compris comme construction sociale de la temporalité économique, permet alors d'analyser l'origine des institutions économiques. Ces dernières, dans le capitalisme, ont la particularité de libérer la futurité économique plutôt que de chercher à la sécuriser le plus possible comme c'était le cas dans les sociétés traditionnelles. Ceci donne au capitalisme une temporalité économique bien spécifique.

Dans une première section, est examinée la capacité respective des diverses approches économiques à rendre compte de l'origine des institutions économiques. Il en ressort que l'approche institutionnaliste de Commons est la plus apte dans cette tâche. La deuxième section met en évidence le rôle crucial de la temporalité quant à l'origine des institutions économiques. Il est alors montré que le principe de futurité proposé à cet effet par Commons est particulièrement pertinent. La troisième section montre comment le 
principe de futurité imprime un genre de causalité spécifique, la causalité institutionnelle, à l'œuvre dans l'activité économique. Enfin, dans une dernière section, il est montré comment les institutions centrales du capitalisme moderne que sont la monnaie de crédit et le capital intangible ne sont compréhensibles qu'à l'aune du principe de futurité.

\section{Institution de l'activité économique}

3 À l'exception de l'institutionnalisme des origines (T. Veblen, J.R. Commons), les théories économiques qui s'intéressent aux institutions économiques postulent généralement l'antériorité logique d'une instance - le Marché, l'infrastructure du Mode de production, le Système social, etc. - sur ces institutions. Celles-ci sont alors posées déductivement en rapport, et souvent en opposition, à cette instance plus "naturelle» (le Marché), plus « historique » (le Mode de production) ou plus « générale » (le Système social)1.

Pour rendre compte de l'origine des institutions économiques, les théories économiques à méthodologie néoclassique mettent de l'avant les «défaillances» du Marché (l'information imparfaite et les interactions stratégiques chez les nouveaux keynésiens ; les coûts de transaction chez les néo-institutionnalistes) ou son "incomplétude " (le manque d'information et de coordination chez les conventionnalistes). D'autres théories, plus sociologiques, invoquent, pour expliquer ces institutions, une contrainte positive de l'« évolution» (le passage du statut au contrat chez H. Spencer 1862) ou normative de " solidarité » (la corporation chez E. Durkheim 1894) nécessaire à l'existence du Marché. D'autres encore présentent les institutions comme des réponses fonctionnelles requises par le Système social (Parsons, 1951). Dans le prolongement de l'analyse marxienne, certaines théories expliquent l'existence des institutions économiques comme des formes d'adaptation du Mode de production capitaliste aux conditions historiques du moment (l'École de la Régulation, Boyer \& Saillard 2010), et ceci selon la variété des circonstances nationales (Hall \& Soskice 2001, Amable 2003). L'une des grandes originalités de l'institutionnalisme, et c'est en cela que son appellation est justifiée, a été de rompre avec la secondarisation analytique des institutions économiques et de placer ces dernières au premier rang de l'analyse économique. Il ne s'agit plus de rendre compte de pourquoi et comment les institutions régulent une instance qui leur serait première (et primitive ?), mais plutôt de comprendre pourquoi et comment les institutions économiques instituent l'activité économique.

Dans cette dernière optique, l'institutionnalisme doit donc reprendre à son fondement la question première de l'intelligibilité de l'institution de l'activité économique. Pour construire une telle théorie économique institutionnaliste il est alors nécessaire d'élaborer une théorie de l'action économique sur laquelle s'appuyer.

6 La théorie dite de l'action rationnelle n'est pas pertinente pour cette tâche (Guerrien \& Benicourt 2008, Keen 2011). Sauf à tomber dans les paradoxes et les incohérences du néoclassicisme, l'institution ne peut être fondée sur les ruses de la raison qui se jouent du pauvre personnage de l'homo ceconomicus. Ajouter des règles externes, les institutions, à la règle interne d'optimisation utilitariste de l'acteur économique est un procédé méthodologique inacceptable. Pour le dire différemment, ajouter des hypothèses auxiliaires ad hoc (les institutions) à une hypothèse principale (l'homo œconomicus) n'a jamais été une bonne méthode pour obtenir des conclusions qui ne peuvent être déduites 
de l'hypothèse principale. Ajouter des normes, règles, conventions, etc. pour "sociologiser" l'homo œconomicus et son contexte d'activité est donc une mauvaise direction.

7 Une autre orientation est, comme le propose T. Veblen (Gislain 2000b) et ses exégètes (Hodgson 2004), de multiplier les règles internes de l'acteur sous formes d'instincts issus de l'évolution adaptative des êtres humains au cours de leur longue histoire. Cette approche, fondée sur un certain darwinisme méthodologique (Gislain 2011b), peut paraître séduisante car elle échappe aux incohérences néoclassiques, mais elle se confronte elle-même à un paradoxe difficilement soutenable. En effet, outre le caractère fort discutable de l'existence de ces fameux « instincts ", ceux-ci se manifestent dans la réalité économique en produisant des résultats, les institutions, ayant un contenu inverse par rapport à la logique de leurs instincts initiateurs. La mentalité dominante de l'acteur institué conduit chez lui à un comportement économique totalement opposé à ses instincts de base. La mentalité «propriétaire » et ses variations secondaires que sont la consommation et le loisir « ostentatoires » sont les institutions économiques dérivées des instincts du travail efficient, de la grégarité et de la curiosité gratuite. Comment cette dérivation-inversion peut-elle se produire ? La lecture de Veblen suggère l'existence d'un quatrième instinct ou propension perturbatrice : la rivalité. Cette dernière, exacerbée par la possibilité d'accaparement du surplus économique issu de l'exercice des trois premiers instincts, pervertirait ces derniers pour les mettre au service de la «comparaison provocante». Les institutions économiques contemporaines seraient ainsi le résultat d'une ruse de l'évolution. Les institutions secondaires, comme la consommation ou le loisir, et tertiaires, comme le sport ou la mode (Veblen 1899), s'ajoutant aux institutions plus évoluées de la propriété, comme le capital financier (Veblen 1904), composeraient une configuration institutionnelle contemporaine dont la logique évolutive serait bien difficile à contrecarrer... sauf à puissamment réactiver la positivité sociale et économique des trois instincts fondamentaux (Veblen 1921)... dont on comprend difficilement pourquoi et comment ils ont pu, eux, ne pas évoluer positivement.

8 La théorie de l'action économique la plus intéressante pour fonder une théorie des institutions nous paraît être celle initiée par J.R. Commons (1934a) ${ }^{2}$. Cette théorie de l'action est bien, comme le propose Max Weber (1921), une théorie de l'action sociale. Elle est une action individuelle socialement construite et son mode de construction sociale réside dans le fait social, au sens durkheimien (Durkheim 1900), qu'elle est instituée. Cette institution est un processus, nous dit Commons (1934a), qui peut être analysé à partir de cinq principes, chacun d'eux permettant de rendre compréhensible une dimension de l'activité économique. Ces principes sont la rareté, l'efficacité, la futurité, la coutume et la souveraineté3. Les deux premiers ont été au fondement des théories économiques passées, le principe d'efficacité pour les théories classiques de Smith à Marx, et le principe de rareté pour les théories utilitaristes et néoclassiques. Ces théories ont été incapables de rendre compte des institutions économiques autrement, comme nous l'avons souligné plus haut, qu'en les secondarisant par rapport à une instance économique plus fondamentale, le Marché, le Mode de production ou le Système. L'activation analytique des trois autres principes est donc nécessaire pour compléter l'analyse institutionnaliste.

9 D'un point de vue logique, le principe de futurité doit être abordé en premier car il constitue, en tant qu'expression du souci humain principal qu'est la crainte concernant l'avenir incertain, l'amorce du comportement humain, et en particulier du comportement 
économique. Ce principe de futurité intègre, dès l'origine de la problématique du comportement économique, la question de la temporalité; question que l'on retrouve traditionnellement à l'occasion de la définition descriptive de l'institution, c'est-à-dire sa dimension temporelle de durée et de pérennité. Mais cette temporalité est généralement peu questionnée en tant que telle. La référence au temps chronologique de la physique est implicitement admise. Or, dans une optique commonsienne, c'est la temporalité ellemême et spécifique à l'activité humaine, en l'occurrence dans sa dimension de futurité, qui est à l'origine de l'institution et, en particulier, de l'institution économique.

\section{De la futurité à l'institution}

10 La prise en compte de la question de la temporalité dans l'activité économique trouve son origine dans un trait fondamental de l'humanité : le souci concernant l'avenir incertain. Cette crainte peut être comprise d'un point de vue biologique, chez tous les êtres vivants, comme étant liée à l'«instinct de survie» (Darwin 1859) ou d'un point de vue spécifiquement humain et phénoménologique comme le "souci de la mort » (Heidegger 1927). Dans tous les cas, l'inscription dans la temporalité « soucieuse » quant à l'avenir est un des fondements essentiels de l'activité humaine.

Une tradition sociologique a parfaitement montré que le «temps » est une construction sociale. Plus encore, ce « temps » fait l'objet de plusieurs types de construction sociale ; le temps chronologique, le temps existentiel, les temps sociaux, etc. ${ }^{4}$. Concernant spécifiquement l'activité économique, la problématique temporelle se fixe initialement sur la crainte de manquer et, en conséquence, sur la volonté d'y échapper. Face à l'inquiétude concernant l'avenir incertain, le futur envisagé, c'est-à-dire le principe de futurité, est au cœur des préoccupations humaines. Il a pour conséquence d'activer deux autres principes qui lui sont corollaires, les principes de rareté et d'efficacité.

12 Le principe de rareté met l'accent sur le manque et est à l'origine des théories économiques de type utilitariste et néoclassique. Ces théories se centrent alors uniquement sur la question des phénomènes $d$ '« échange » comme instance de circulation de la rareté et sur son idéaltype qu'est le Marché. De son côté le principe d'efficacité concerne la volonté d'éliminer la rareté et est à l'origine des théories classiques. Ces théories privilégient alors l'instance de la production comme mode de résolution du problème de la rareté. Commons qualifie respectivement ces deux types d'approche de théorie de la rareté et théorie de l'abondance. Dans les deux cas, le principe antécédent de futurité disparaît de l'analyse économique au profit d'une fixation sur l'une de ces deux conséquences, la rareté et l'efficacité. En conservant la primauté du principe de futurité, ce qui n'exclut pas la réalité des deux autres principes conséquents mais en les abordant alors subséquemment différemment ${ }^{5}$, une autre analyse est possible dont Commons nous en offre les éléments fondamentaux.

13 Le principe de futurité étant maintenu à l'origine de l'analyse, une autre réponse peut être apportée grâce à une appréhension pragmatiste de l'activité humaine. Et c'est effectivement cette orientation que prend Commons en adhérant explicitement à la philosophie pragmatiste (Commons 1934a, p. 150-157), en particulier celle de Charles Sanders Peirce (1931-1958) et John Dewey (1972-1985) ${ }^{6}$. En effet, pour le pragmatisme, l'activité humaine se caractérise, face à un problème, en l'occurrence ici celui de la crainte de l'avenir incertain, par la mobilisation de l'intelligence humaine sur le mode de la résolution de problème: l'enquête (Dewey 1938). Selon une logique d'apprentissage 
fondée sur l'abduction, c'est-à-dire l'itération circulaire induction-déduction jusqu'à une solution satisfaisante (Tool 1994), l'expérience humaine conduit à des résultats que Commons appelle des hypothèses habituelles (1934a, p.697-714). Ces dernières, lorsqu'elles sont partagées par un groupement humain, un groupe actif (going concern) ${ }^{7}$, deviennent des habitudes sociales, des pratiques comportementales normales dans telle ou telle situation. Le plus souvent, ces pratiques sociales habituelles existent avant la naissance de l'individu. Le processus de socialisation de ce dernier consiste d'ailleurs essentiellement à l'apprentissage transactionnel de ces habitudes sociales (Mead 1934). Inscrites dans la durée, celles-ci sont ce que Commons appelle des coutumes, des actions collectives inorganisées ${ }^{8}$. Et ces coutumes, formes primitives des institutions, ont pour vocation essentielle de sécuriser la futurité des membres du groupe actif. En anthropologie, et en particulier celle du début du siècle ayant influencé Commons, la coutume est qualifiée de mœurs, usage, manière, ou plus généralement de "folklore " (Sumner 1906). En sociologie, les notions de règle, norme ou convention sont traditionnellement utilisées pour qualifier un mode comportemental qui s'inscrit dans la durée. Commons choisit le concept de coutume, et plus généralement le principe de coutume $^{9}$ pour caractériser toutes les formes de régularité et de récurrence dans les comportements, c'est-à-dire tout ce qui structure la futurité des transacteurs ${ }^{10}$, les guide pour se projeter dans l'avenir. La coutume est une norme comportementale qui le plus souvent "va sans dire ", s'impose à chacun communément et dont chacun s'attend à ce que les autres s'y conforment. C'est une forme primitive d'intentionnalité collective.

Dans le domaine de l'activité économique, ces coutumes cherchent à lutter contre la rareté relative des biens et services disponibles dans l'avenir et peuvent prendre de nombreuses formes. L'anthropologie économique nous révèle une multitude de solutions tant au point de vue de l'organisation de la production (formes de division du travail) que de celui de la circulation des objets économiques (don-contredon, échange aveugle, crédit, négoce, etc.) comme moyens de réduction de la rareté, c'est-à-dire de sécurisation de la futurité. Toutes ces formes économiques sont initialement instituées comme coutumes et n'ont donc rien de "naturelles » comme le Marché (Graeber 2011) ou de «nécessaires» comme tel ou tel type d'organisation du travail (Clastres 1974). Les solutions aux principes de rareté et d'efficacité, c'est-à-dire la réalité de l'activité économique, sont initialement des coutumes instituées issues de l'apprentissage expérimental des groupes actifs.

15 À chaque moment de l'histoire économique émergent de nouvelles pratiques, éprouvées expérimentalement, qui s'imposent d'abord comme solution coutumière s'instituant progressivement comme normes de l'activité économique ${ }^{11}$. Celles-ci peuvent être catégorisées sous divers concepts par certaines théories socioéconomiques, comme la théorie des "conventions" (Revue économique 1989) ou celle des "communs» (Ostrom 1990), il n'en demeure pas moins, que ce sont avant tout des coutumes au sens pragmatiste commonsien du terme.

Ces formes inorganisées d'action collective que sont les coutumes peuvent perdurer longtemps tant qu'elles répondent adéquatement, comme pratiques économiques socialement partagées, à la résolution de problèmes concernant l'avenir incertain. Que ce soit se serrer la main pour conclure une transaction à terme, l'usage de l'endos d'une carte à jouer pour inscrire une dette, l'adoption d'un talisman comme monnaie, les rythmes de travail dans la production, le paiement par chèque, et les exemples pourraient 
être multipliés à l'infini, toutes ces coutumes sont des institutions économiques qui régissent l'activité économique.

Toutefois, certaines de ces coutumes peuvent à un certain moment être l'objet d'une contestation ou à tout le moins l'objet d'un besoin de formalisation plus explicite. Dans tous les cas, le fait est que la coutume ne peut être maintenue telle quelle par les transacteurs, c'est-à-dire par les acteurs individuels soumis coutumièrement à la même action collective inorganisée. La coutume doit évoluer selon un processus de sélection $\operatorname{artificielle}^{12}$. Les conséquences attendues de cette coutume sont maintenant perçues par ces transacteurs soit comme inacceptables, soit comme ayant un impact allant au-delà des seuls habituels transacteurs régis par cette coutume.

possibles : une nouvelle coutume vient se substituer à l'ancienne ; la coutume contestée fait l'objet d'une réorganisation formelle et les membres du groupe actif qu'elle régit sont maintenant soumis à une action collective organisée propre au groupe actif ; la coutume mise en cause est l'objet d'un jugement par une instance « autorisée » supérieure au groupe actif concerné.

La première situation correspond au processus évolutionnaire des coutumes se succédant selon les apprentissages continus des transacteurs. Par exemple, la coutume de validité des transactions par la parole donnée peut être remplacée par la nouvelle coutume de la présence d'un témoin communément accepté et pouvant ainsi témoigner de la validité de la transaction. La deuxième situation émane d'une réflexion des transacteurs d'un groupe actif, le plus souvent d'ailleurs de ses dirigeants, sur l'efficacité de celui-ci dans sa lutte contre la rareté. Il s'agit, par exemple, de formaliser des règles opérantes de conduite internes à l'organisation de la production. L'action collective passe alors de la forme coutumière inorganisée à la forme plus évoluée organisée. Par exemple, ce peut être le passage d'une activité productive coutumière de type artisanal, où chacun des transacteurs sait ce qu'il a à faire selon sa place dans le processus de production, à une organisation productive de type manufacture avec ses règlements et directions hiérarchiques. Ce peut être aussi la répartition des pertes et bénéfices d'une activité entre les transacteurs ou les membres d'un même groupe actif. Par ces opérations, apparait le principe de souveraineté s'appliquant à l'interne du groupe actif ou entre les transacteurs. Dans les exemples retenus, naît alors la gouvernance d'entreprise, cette dernière étant maintenant formellement instituée. La troisième situation concerne l'évolution de la coutume vers une remise en cause interne ou externe au groupe actif. Sans solution de réorganisation comme dans le deuxième cas, la coutume incriminée est alors soumise à une instance supérieure « autorisée » qui devra trancher sur l'avenir de cette coutume. Le principe de souveraineté s'applique maintenant dans ses versions juridique ou politique. Par exemple, si une pratique commerciale coutumière est remise en cause par les commerçants ou les clients, un juge ou une autorité politique peut statuer soit sur sa validité et ainsi l'instituer légalement, soit sur son illégalité et la bannir dorénavant des pratiques commerciales. Une coutume commerciale disparait ainsi ou devient une institution formelle de l'activité commerciale.

Dans le second cas où la coutume a un impact qui s'étend au-delà des seuls habituels transacteurs régis par cette coutume, cette dernière devient un enjeu public au sens que John Dewey (1927) donne au terme de "public », c'est-à-dire tous ceux qui sont concernés par les conséquences d'une transaction. Ce type de problème public appelle des solutions politiques et juridiques de « réforme » des institutions. 
21 De nombreuses situations correspondent à ce cas. Par exemple, l'interdiction par le nouveau régime politique de la Révolution française des corporations de métier et de toute forme de coalition ouvrière ou patronale a conduit à l'institution du " marché du travail ». En prenant cette décision les membres de la Constituante ont activé le principe de souveraineté pour instituer un nouveau régime économique de la relation d'emploi. La nouvelle école d'histoire économique néo-institutionnaliste (North 1990) relate de nombreux exemples d'institutionnalisation économique, en particulier concernant les droits de propriété, mais leurs explications cherchant à justifier ces faits historiques sont, comme en général pour les théories d'inspiration néoclassique, fondée sur un raisonnement circulaire inacceptable. Toute nouvelle institution économique novatrice est expliquée par sa miraculeuse adéquation avec la logique de l'intérêt de l'homo œconomicus... constatée a posteriori, une fois établie cette nouvelle institution.

En revanche, les analyses commonsiennes défendent plutôt une explication évolutionnaire où le processus d'établissement d'une nouvelle institution économique est rendu intelligible par le principe de souveraineté s'appliquant dans un contexte historique toujours spécifique. Par exemple, selon Commons, le droit de propriété privée moderne est né au dix-septième siècle anglais alors que les forces politiques des bourgeois commerçants étaient en mesure d'imposer aux pouvoirs seigneuriaux cette nouvelle institution économique (Commons 1924). De la sorte, les commerçants anglais se mettaient à l'abri des aléas des pouvoirs discrétionnaires des seigneurs. Ces commerçants sécurisaient ainsi leur futurité grâce à l'institution de leurs droits de propriété.

Dès ses premiers écrits (Commons 1893) et tout au long de son œuvre, Commons défend l'idée que le principe de souveraineté est au service de la sécurisation de la futurité de certaines classes sociales, que ce soit en général dans la société, comme dans le cas précédent, ou au niveau plus particulier de "privilèges » économiques accordés à certains groupes actifs, la situation la plus ordinaire étant l'institution d'un rationnement de l'offre (monopole légal, exclusivité, brevet, charge, office, copyright, etc.). Le principe de souveraineté agit alors comme un distributeur différentiel de rentes de situation (goodwill), c'est-à-dire de futurités sécurisée au profit de certains transacteurs et, consécutivement, souvent au détriment d'autres. À cet égard, le projet politique général de Commons, un genre de social-démocratie à l'américaine et dont quelques composantes ont été instituées par le New Deal (da Costa 2010), est justement d'instituer la sécurisation de la relation d'emploi en donnant de nouveaux droits aux travailleurs réduisant l'incertitude de leur futurité comme cela avait été le cas pour les commerçants anglais trois siècles plus tôt $\mathrm{t}^{13}$.

\section{La causalité institutionnelle}

La conception pragmatiste du temps dans l'activité économique proposée par Commons lui a permis de construire le concept de futurité et de l'appliquer comme principe à l'analyse économique en mettant en évidence le rôle crucial de la causalité institutionnelle (Gislain 2002, 2011a).

En effet, pour Commons, "Pragmatism is Futurity" (1934a, 152). Il en arrive à cette affirmation en appliquant la maxime générale de la philosophie pragmatiste à la question de la temporalité de l'activité économique. Cette maxime est proposée par C.S. Peirce dans les termes suivants: "Considérer quels sont les effets pratiques que nous pensons 
pouvoir être produits par l'objet de notre conception. La conception de tous ces effets est la conception complète de l'objet » (Peirce 1878, p. 297 ; Commons 1934a, p. 152). Inscrits dans la temporalité de l'activité, les éléments de cette maxime peuvent se comprendre de la façon suivante. La « conception de tous les effets pratiques que nous pensons pouvoir être produits par l'objet de notre conception » est la futurité telle que la conçoit tout transacteur, c'est-à-dire tout ce qu'il pense que son activité implique pratiquement dans l'avenir. Dans ces conditions, la « conception de tous ces effets est la conception complète de l'objet» signifie que la futurité, en tant que (troisième) ${ }^{14}$ monde constitué de l'ensemble des effets attendus de son activité, est à partir de quoi le transacteur conçoit son activité15. La temporalité spécifique de l'activité a pour objet et consiste à se projeter dans la futurité ${ }^{16}$. Commons résume simplement ce fait par cet apparent paradoxe : «les hommes vivent dans le futur mais agissent dans le présent " (1934a, p. 58, p. 84). Ainsi comprise, la construction sociale de la temporalité de l'activité rompt significativement avec d'autres types de construction sociale de la temporalité ${ }^{17}$, et en particulier avec la construction sociale traditionnelle du temps physique ${ }^{18}$. Dans cette dernière, la logique temporelle est soumise à un principe de causalité de type passé- présent-futur ; la flèche temporelle et la causalité à l'œuvre dans le temps chronologique ont la même orientation. En revanche, dans l'activité soumise au tropisme de la futurité, la logique à l'œuvre, qui est une logique volitionnelle plutôt que déterministe, a une causalité orientée inversement à celle de la causalité temporelle physique-chronologique. Dès lors, concernant l'activité humaine, « cela change l'idée de causalité. Cela place définitivement la causalité dans le futur plutôt que dans le passé » (1934a, p. 7) et, d'un point de vue logique, alors l'« effet précède sa cause » (1939a, p. 130).

Dans ces conditions, le passé ne perd pas pour autant de son importance mais il est construit différemment et a un impact plus putatif que déterministe. Le passé pertinent retenu par le transacteur est celui qui l'informe présentement sur sa futurité et donc sur les possibilités futures d'activité et ses conséquences. La chaîne causale complète de l'activité n'est plus alors, comme dans le monde physique, une flèche orientée passéprésent-futur mais plutôt une boucle où au présent est sélectionné un passé pertinent pour envisager la futurité qui conditionnera l'activité présente. Les séquences causales sont maintenant orientées selon le cercle : présent-passé-futurité-présent. La futurité est l'horizon de l'activité présente à partir que ce que présentement le passé nous informe sur la futurité. Selon cette temporalité, comme le passé et le présent, la futurité existe actuellement, pas le futur qui demeure inconnu ${ }^{19}$. D'un point de vue évolutif, les perspectives d'activité sont donc sans cesse actualisées, la futurité étant ainsi ambulatoire. Chaque activité présente (expérience) modifie le passé pertinent (conséquences constatées) sur la base duquel est conçue la futurité comme perspectives d'activité (anticipations raisonnables) ${ }^{20}$.

La notion d'anticipation, qu'utilise souvent Commons, prend alors un sens bien spécifique. Il ne s'agit plus de chercher à découvrir le futur, qui est de toute façon radicalement incertain ${ }^{21}$, mais plutôt de l'appréhender « en connaissance de cause » et ces dernières sont précisément le contenu de la futurité. Et ce contenu n'est pas le futur mais ce que nous en savons d'expérience et par apprentissage. Le principe de futurité relève donc bien de la philosophie pragmatiste appliquée à l'activité humaine, et en général à «l'homme (qui) est un être dans le temps» (Commons 1935, p. 477).

Sur la base de cette conception bien spécifique de la temporalité où, en général, le «principe de futurité domine l'activité» (1934a, p. 84), Commons en conclut tout 
logiquement que toute science humaine doit être une «science de la futurité » (1943a, p. 84), en particulier la science économique car «la futurité est le principe le plus important de l'économie » $(1934 \mathrm{~b}, \mathrm{p} .125)^{22}$.

Pour Commons, le principe de futurité, conjugué aux quatre autres principes à l'œuvre dans l'économie, impose à l'analyse économique, outre d'être multicausale en raison de l'action des cinq principes, la prise en compte d'une causalité institutionnelle orientée selon le cercle temporel identifié précédemment. L'analyse économique traditionnelle s'en trouve alors radicalement modifiée. Au niveau de la théorie de l'action économique, l'activité doit être comprise en rapport à une actualité définie par la futurité.

L'être humain agit dans le présent, en vue de ce qu'il anticipe qui arrivera dans le futur; de la sorte, la théorie de la causalité qui devrait être appliquée en théorie économique est la théorie de l'anticipation. Son acte présent est causé par ce qu'il anticipe dans le futur. En d'autres termes, dans toutes ces affaires qui nous concernent, la causalité n'est pas dans le passé mais dans le futur (1929a, p. 61).

Ceci modifie aussi radicalement l'objet de l'analyse économique car on :

[...] trouve toujours la futurité, non dans la production et la consommation, mais dans les persuasions et coercitions des transactions de marchandage, les ordres et obéissances des transactions de direction, les argumentaires et plaidoyers des transactions de répartition, qui détermineront ultimement la production et la consommation. Dans ces négociations et décisions, qui sont l'essence de l'économie institutionnelle, ce sont toujours la production future et la consommation future qui en sont l'enjeu, car les négociations déterminent le contrôle légal qui devra précéder le contrôle physique (1934a, p. 7).

31 Dans le passé et le présent, les «objets économiques » sont des marchandises, objets de l'analyse économique traditionnelle fondée sur les principes de rareté et d'efficacité. Lorsque, comme il se doit, la futurité est prise en considération pour rendre compte de l'activité dans sa temporalité, ce sont les propriétés, et plus spécifiquement les droits de propriété, qui deviennent les objets pertinents de l'économie institutionnaliste ${ }^{23}$. Ainsi pour Commons :

Les biens matériels sont de simples accumulations physiques du passé jusqu'au point présent du temps. Ils n'existent pas en tant que propriété ou valeur pour l'être humain jusqu'à ce que, à partir du point présent du temps, une futurité leur soit attribuée. Car la propriété (propriété corporelle ou incorporelle) est toujours un droit présent à un usage ou une vente futurs de biens matériels, immédiatement ou reportés dans le futur. Les biens matériels, en tant que simples existences physiques, sont toujours dans le passé. Ils n'ont en eux-mêmes pas de futurité. Mais la propriété et l'évaluation de ces biens matériels regardent toujours vers le futur. Les deux sont séparés par un point mobile du temps, le présent, instant où les biens matériels finissent, parce qu'ils n'ont pas d'anticipations; mais leur propriété et valeur commencent à ce point mobile du temps, le présent, parce qu'ils sont l'objet des anticipations humaines (1934a, p. 406-407)

En d'autres termes, l'analyse économique se doit nécessairement, du fait de son objet même, d'être une analyse possédant une dimension temporelle incontournable mais, de plus, une temporalité dont la causalité à l'œuvre est bien spécifique. Cette causalité, différente de la causalité chronologique des sciences naturelles, a deux grandes caractéristiques. D'une part, elle est orientée selon un cercle procédant à l'actualisation de l'activité, comme nous l'avons déjà souligné. D'autre part, elle donne aux institutions, qui structurent la futurité, un pouvoir causal sur l'activité. Les transacteurs orientent leurs actions par rapport à ce que leur offrent les institutions comme expressions de leur futurité. L'activité des transacteurs est ordonnée ${ }^{24}$ par une intentionnalité collective, 
c'est-à-dire une futurité significative commune ${ }^{25}$, dont l'expression concrète est l'action collective $^{26}$. En somme, pour l'analyse économique institutionnaliste prenant en considération le rôle crucial du principe de futurité dans l'activité économique, « le temps est un concept tout à fait institutionnel » (Commons 1934a, p. 642).

\section{Futurité et institutions du capitalisme}

certaines catégories de transacteurs, le plus souvent réciproquement au détriment d'autres catégories. En régime capitaliste, on retrouve cette même logique d'institution codifiée par les instances juridiques, accumulée dans la jurisprudence ou présente dans la connaissance commune. Dans son ouvrage Legal Foundations of Capitalism (1924), Commons a analysé à la fois le processus historique d'apparition de ces institutions et leur contenu contraignant, libératoire ou potentiel ${ }^{27}$, en particulier concernant les droits de propriété et les formes de persuasion ou de coercition économiques. Tout au long de son œuvre, Commons, comme professeur de relations industrielles et réformateur en matière de politique publique, a analysé les institutions de la relation d'emploi sous le capitalisme. Pour Commons, ces institutions s'inscrivent dans l'histoire longue de l'exploitation du travail avec les spécificités historiques du salariat moderne. Cependant, il pense qu'à l'image de la sécurisation contemporaine de la futurité du capital industriel, en particulier de l'investissement productif, il est possible de réformer les institutions du salariat dans le but de mieux sécuriser la futurité des travailleurs. La liste est longue des réformes que propose Commons dans cette optique d'institution d'un État Social. Aux États-Unis, certaines de ces réformes, directement ou sous l'inspiration de Commons verront le jour de son vivant, notamment à l'occasion du New Deal (assurance accident du travail, assurance chômage, reconnaissance du droit syndical, assistance sociale, etc.) ; d'autres devront attendre le siècle suivant (assurance maladie universelle, congés parentaux, etc.), ou attendent encore leur institution (droit au logement, sécurisation d'un revenu décent, etc.). Sous ces aspects institutionnels, le capitalisme historique est donc dans la lignée de l'histoire longue des sociétés humaines où, traditionnellement, une lutte, arbitrée par le principe de souveraineté, s'opère sur la distribution des pouvoirs économiques. Ces pouvoirs prennent essentiellement la forme de la sécurisation de la futurité pour certains transacteurs, le plus souvent au détriment réciproque d'autres transacteurs. C'est dans cette optique de lutte pour les pouvoirs de sécurisation de la futurité que s'inscrit la problématique réformiste de Commons. Il s'agit de réformer certaines institutions du capitalisme pour le rendre plus « raisonnable ». En ce sens, on peut dire que le projet commonsien de «capitalisme raisonnable » est un programme politique de type social-démocrate. Peut-être le seul que les États-Unis aient produit dans leur histoire.

aspect de la causalité institutionnelle appliquée au capitalisme contemporain, et analysé par Commons, est beaucoup plus original par rapport à l'histoire longue des sociétés humaines. Alors qu'au cours de cette dernière, traditionnellement et comme cela a été souligné ci-dessus pour un certain aspect du capitalisme aussi, l'institutionnalisation économique est un processus de lutte pour la sécurisation des futurités respectives des transacteurs, le capitalisme moderne cherche plutôt à «libérer» ces futurités, c'est-à- 
dire à augmenter le degré d'incertitude quant au futur. Cette libéralisation offre autant d'opportunités au déploiement du capital dans sa forme plénière : le capital intangible. Cet avènement $d u$ règne $d u$ capital intangible, la forme la plus capitalisable financièrement des objets de l'économie que sont les droits de propriété sur l'usage futur des biens et services, est initialement rendu possible par la dimension fondamentalement monétaire de l'activité économique, la monnaie étant elle-même essentiellement une monnaie de crédit. Ce sont ces deux dimensions du capitalisme "libéré", ce que Commons nomme le capitalisme bancaire, la monnaie de crédit et le capital intangible, auxquelles nous allons nous intéresser maintenant.

\subsection{Monnaie de crédit}

La théorie monétaire de Commons est en rupture radicale avec les approches orthodoxes classique et néoclassique ${ }^{28}$. Il élabore sa théorie à partir d'une discussion sur les théories monétaires plus ou moins hétérodoxes de H.D. MacLeod (1856, 1867), K. Wicksell (1898), G. Cassell (1903), G.F. Knapp (1905), R.G. Hawtrey (1919), I. Fisher $(1906,1932)$ et J.M. Keynes (1930), pour prendre les principales.

Commons a une conception purement nominale de la monnaie ${ }^{29}$, c'est-à-dire sans référence à ce que serait sa valeur " réelle ", que ce soit une valeur-travail ou une valeurutilités $^{30}$. La monnaie est pour lui l'institution qui permet de nommer économiquement, c'est-à-dire de quantifier par un prix dans une futurité proche ou éloignée, un droit sur une propriété, c'est-à-dire sur l'usage futur d'un bien ou d'un service ${ }^{31}$. La monnaie est ce qui permet socialement d'inscrire dans le temps plus ou moins long une quantité de droit de propriété. La monnaie est ainsi comprise comme une "unité de mesure purement artificielle, collective et "nominale"» (1934a, p. 213). Elle "mesure la rareté propriétarienne » c'est-à-dire «le pouvoir économique, l'égalité des opportunités, la compétition loyale et déloyale, les valeurs raisonnables et déraisonnables dans un contexte régi par la politique, les lois et d'autres formes d'action collective» (1934a, p. 213). Cette valeur-rareté propriétarienne relève donc «du pouvoir collectif sur les individus que nous appelons des institutions. L'une de ces institutions est la monnaie entendue au sens d'instrument collectif pour la création, la négociation et la libération des dettes par les individus » (134a, p. 278). La valeur propriétarienne, qu'est le prix relatif constaté, synthétise, et est le résultat, de l'action conjuguée des cinq principes à l'œuvre dans l'activité économique : rareté, efficacité, souveraineté, coutume et futurité. L'action de ce dernier principe est ce qui permet, particulièrement, au prix d'intégrer la dimension temporelle de l'activité économique.

La monnaie a elle-même une valeur-rareté propriétarienne "qui varie en fonction inverse de son pouvoir d'achat moyen» (1934a, p. 262). Dans ces conditions, les prix relatifs que mesure la monnaie sont des quantités de même nature, sans dimension physique, que les températures relatives que mesure un thermomètre ${ }^{32}$, avec cette différence que le référentiel de l'unité monétaire doit être corrigé de ses variations temporelles de pouvoir d'achat ${ }^{33}$.

39 Pour Commons, le caractère nominal de la monnaie est lié sa fonction première, logiquement et historiquement pour reprendre la distinction de R.G. Hawtrey (1919), d'unité de compte par rapport à sa fonction seconde de moyen de paiement libératoire des dettes, de négociabilité de la dette et de réserve de valeur ${ }^{34}$. Cette origine nominale de la monnaie se perpétue jusqu'à nos jours au travers d'une «longue période de mise au 
point de la négociabilité de la dette » et qui a fini par « convertir de simples anticipations monétaires en monnaie elle-même » (1934a, p. 393). Concernant les sociétés modernes, Commons s'inscrit dans la perspective de la conception chartaliste de la monnaie de G.F. Knapp (1905), selon laquelle la monnaie est une institution politique dont l'État décide, en dernier ressort, la légalité dans les transactions. La monnaie est un attribut de la souveraineté politique de l'État.

Cet aspect «public» de la monnaie est aussi conforme à la conception générale du « public » proposée par J. Dewey (1927). Ce dernier caractérise ce qui est de l'ordre du "public» comme étant tous les effets d'une transaction qui dépassent les seuls transacteurs et font donc "problème " pour la collectivité. L'instance politique est alors convoquée, sous la pression des opinions publiques se saisissant de ce type de problème, à contrôler ces effets selon ce qu'elle pense être l'intérêt commun. Pour Commons, toute transaction économique a effectivement cette caractéristique et requiert donc une tierce partie, invertie de la souveraineté, garante d'une certaine idée du bien public. Un « juge », ou toute autorité représentative de la légitimité souveraine, s'acquitte en général de cette fonction. En matière monétaire, le politique définit donc la monnaie comme relevant du " public » (au sens de J. Dewey) et en détermine les attributs légaux (cours, convertibilité, etc.). C'est ce qui fait aussi que la monnaie est par définition une institution ${ }^{35}$ et non, comme le voudrait la théorie monétaire orthodoxe, une simple marchandise fonctionnelle facilitant les échanges ${ }^{36}$.

41 La forme plénière de la monnaie est, pour Commons, la monnaie de crédit, c'est-à-dire une monnaie émanant de la nature fondamentale de la relation transactionnelle entre groupes actifs, que ces groupes soient réduits à une seule personne ou étendus à une grande organisation. Cette relation est une transaction de marchandage dans laquelle une double dette engage les transacteurs : une dette de livraison pour le vendeur et une dette de paiement pour l'acheteur. Les deux dettes s'inscrivant dans une futurité plus ou moins proche ou éloignée, il est donc nécessaire d'en nommer respectivement les teneurs et leurs quantités. Pour la dette de livraison, la teneur et la quantité ont des mesures physiques. En revanche, pour la dette de paiement la teneur est purement nominale, une unité de compte monétaire, et la quantité est un nombre chiffré de cette unité, un prix. Dans la transaction, la livraison et le paiement peuvent être respectivement plus ou moins retardés et donner lieu à des reconnaissances de dette à terme. Ces dernières, ainsi projetées dans la futurité, et cela d'autant plus qu'elles sont négociables, peuvent être soit escomptées, c'est-à-dire être converties en paiement présent moyennant un intérêt défalqué, soit utilisées comme moyen de paiement, aussi défalqué d'un intérêt, dans une autre transaction. Ces deux possibilités de "papiers " inscrites dans la futurité peuvent elles-mêmes donner lieu à une série temporelle de réescomptes et ainsi se multiplier. Cela correspond à "convertir de simples anticipations monétaires en monnaie elle-même " (1943a, p. 393). La futurité se peuple alors d'une multitude de «papiers » originaires de dettes, c'est-à-dire "de "quantités économiques" qui se différencient des quantités matérielles du seul fait qu'elles n'existent que dans le futur » (1934a, p. 412). Ainsi, pour Commons, «si la science économique doit reposer sur la dette, alors nous disposons d'une quantité économique universelle sur laquelle la fonder. Lorsque sa valeur actualisée est mesurée en dollars ou en cents, une dette est tout aussi quantitative qu'une chose physique lorsque le blé est mesuré en boisseaux et l'eau en pintes » (1934a, p. 419). Ces "papiers » sont plus ou moins « actifs", et la frontière entre ces actifs et la monnaie au sens strict n'est qu'une question de «liquidité », c'est-à-dire d'éloignement et de 
négociabilité dans la futurité. Dans le présent, la monnaie apparaît comme "liquide » dans son rôle libératoire de la dette, et elle est « active » dans la futurité et son processus de négociabilité des dettes dans le temps, ce sont alors des actifs. Cette " activité » est au cœur du processus monétaire qui sous-tend l'un des aspects fondamentaux du capitalisme : la production d'argent avec de l'argent au cours du temps.

En abordant la relation économique comme une relation entre transacteurs portant sur une double dette transactionnelle et non comme un échange d'équivalents entre marchandises comme dans la tradition économique orthodoxe, Commons place ainsi la monnaie au fondement de l'activité économique. La monnaie n'est plus comprise comme un équivalent-valeur général « facilitant » les " échanges » de " marchandises », mais elle est plutôt appréhendée comme l'expression nominale de quantité de dettes issues de transactions. La monnaie assure la négociabilité des dettes et permet aux transacteurs de se projeter dans la futurité en limitant plus ou moins l'incertitude liée au futur. Elle fonctionne alors soit comme moyen de paiement (libération de la dette) soit comme actif (négociabilité et transférabilité de la dette). C'est en plaçant ainsi la figure de la dette plutôt que celle de l'échange au fondement de l'activité économique que Commons en arrive à cette théorie monétaire très hétérodoxe ${ }^{37}$. Cette dernière atteint son objectif explicatif le plus pertinent lorsque Commons introduit un type de transacteur bien particulier car spécialisé dans le commerce de l'argent : la banque.

Appréhender l'activité économique à partir de la dette plutôt qu'à partir de l'échange entraîne que la monnaie, permettant la libération ou la négociabilité des dettes, doit être essentiellement comprise comme instrument de crédit. Commons reconnaît explicitement que le premier à initier ce renversement analytique est H.D. MacLeod (1856, 1867), pour qui «la futurité prend objectivement corps dans une "quantité économique" présente, le crédit, l'équivalent de la dette " (1934a, p. 398). Dès que la monnaie de compte existe, et il est fort probable que ce soit pour cela qu'elle soit effectivement apparue dans l'histoire (Commons 1934a, p. 392; Testart 2001; Graeber 2011), le crédit inscrit dans la dette s'exprime en une quantité monétaire plutôt qu'en une quantité physique. Dès lors, une fois légale, la monnaie elle-même peut être l'objet du crédit et devenir alors pleinement monnaie de crédit et crédit en monnaie.

Dans l'économie contemporaine que Commons qualifie de capitalisme bancaire (1934a, p.122), tout détenteur d'une quelconque caution plus ou moins solvable, tangible ou intangible, peut obtenir d'une banque un crédit, c'est-à-dire, d'une part, une somme de monnaie créée ex nihilo par la banque, inscrit à son passif et mise à la disposition de l'emprunteur, d'autre part, une créance sur l'emprunteur du même montant plus les intérêts, mise à l'actif de la banque ou escomptée par celle-ci auprès d'une autre institution financière, cette dernière pouvant à son tour réescompter cette créance ou la titriser, et ainsi de suite tant que l'escompte, la part d'intérêt qui reste attaché à la créance, est profitable pour un réescompteur éventuel. Ce processus, cautionné par le prêteur en dernier ressort qu'est la banque centrale, est celui de la création endogène de monnaie : la monnaie nouvellement créée a pour origine le crédit offert par les banques aux emprunteurs solvables, c'est-à-dire dont la dette a des chances raisonnables, aux yeux des banques, d'être honorée dans le futur. C'est le caractère plus moins sécuritaire de la futurité des emprunteurs qui déterminent leur solvabilité, leur accès au crédit et donc à de la monnaie neuve. Ce processus de création monétaire endogène, laissé à la discrétion des banques selon une réglementation plus ou moins contraignante (taux de 
réserve obligatoire, taux d'escompte, etc.), est, pour Commons, à l'origine des fluctuations monétaires, en particulier les tensions déflationnistes ou inflationnistes ${ }^{38}$.

Sur la base de cette conception de la monnaie endogène et de ses caractéristiques, Commons développe sa conception de la politique monétaire ${ }^{39}$, essentiellement orientée vers la stabilisation monétaire grâce à une politique volontariste par la banque centrale du contrôle du crédit bancaire, notamment par la méthode de l'open market, nouvelle à l'époque où les banques centrales se cantonnaient simplement à une politique de taux d'escompte et, plus récemment, de mutuellisation des réserves de liquidités (d'où le terme de Reserve pour ces banques centrales aux États-Unis).

En somme, on peut traduire la conception monétaire de Commons en utilisant le concept de différance proposé par J. Derrida (1967). D'une part, la monnaie permet de différencier les objets économiques grâce aux prix relatifs, c'est-à-dire par une quantification spécifique au domaine économique. La monnaie donne ainsi une forme sociale propre à ces objets économiques et, de la sorte, en permet la subsumption en des quantités abstraites (Simmel 1900). D'autre part, la monnaie permet de différer l'activité selon deux modes de temporalisation spécifiques au domaine économique. Premièrement, la monnaie permet de projeter l'activité économique du passé vers la futurité grâce à la négociabilité des dettes. Cet aspect est accentué par la monnaie de crédit pouvant se muter en actifs chiffrés en monnaie actuelle. Deuxièmement, la monnaie ouvre un devenir à l'activité économique sous la forme de promesses de revenus dans la futurité quantifiées monétairement par l'actualisation de ces revenus. La monnaie permet de rendre quantifiable actuellement la futurité et ainsi, en quelque sorte, de l'objectiver. Commons propose le concept de capital intangible pour analyser la forme plénière de la temporalisation économique permise par l'institution de la monnaie.

\subsection{Capital intangible}

L'analyse monétaire de Commons lui permet d'aborder la question du capital à partir des types de temporalisation économique qui lui sont liés. De la sorte, Commons différencie le capital corporel, le capital incorporel et de capital intangible. Le capital corporel est une propriété physique actuelle et émane du passé ; sa valeur correspond à son prix actuel. En revanche, les deux autres sortes de capital s'inscrivent dans la futurité mais sont actualisées différemment. Le capital intangible a pour origine «le futur revenu tiré des ventes - à savoir, la propriété intangible - qui est à distinguer du futur revenu provenant du règlement de dettes - à savoir la propriété incorporelle»(1934a, p.419), cette dernière étant à l'origine du capital incorporel. Pour bien faire cette différenciation entre ces deux sortes de capital, incorporel et intangible, inscrites dans la futurité, Commons (1934, p. 520-521) met en évidence deux dimensions de la temporalité de la futurité, offrant respectivement deux formes différentes de rentabilité et d'actualisation monétaires.

L'une de ces dimensions renvoie à la futurité en tant que laps de temps dans le futur, c'est-à-dire « un intervalle anticipé entre un point présent du temps et un point futur du temps » (1934a, p. 407). Le capital qui s'engage dans cette dimension de la futurité, grâce notamment à la monnaie qui permet la négociabilité des dettes et le crédit, est en situation d'attente (waiting), c'est-à-dire une remise à plus tard (postponement) de sa rémunération. La forme économique de cette dernière est alors l'intérêt. L'actualisation monétaire de ce capital incorporel est le montant de la créance (dette) plus (moins) 
l'intérêt selon que l'on est créancier (débiteur). La vie active de ce capital dans la futurité prend la forme du réescompte ou, plus récemment, de la titrisation financière.

L'autre dimension temporelle de la futurité est relative au flux du temps, c'est alors « une succession anticipée d'événements » (1934a, p. 407). Le temps ponctuel se répète tant que le capital engagé dans cette voie perpétue son engagement, sous forme productive ou financière. Dans cette situation, la futurité est le lieu d'une prise de risque (risking) car la prévision (forecasting) qui la concerne est plus ou moins sécuritaire. La rémunération de ce capital est alors le profit. L'actualisation monétaire de ce capital est la somme actualisée de ses revenus anticipés. La vie active de ce capital dans la futurité prend la forme moderne d'un titre financier (action, obligation, et autres « dérivés »). La qualité de ce capital, en tant que capital intangible, réside surtout dans le contrôle d'une situation économique favorable quant à l'anticipation de revenus futurs, c'est-à-dire une rente de situation (good-will).

50 La distinction entre ces deux dimensions de la futuritéte, laps et flux du temps, est analytique et permet surtout de différencier ce qui caractérise plus spécifiquement les deux sortes de capital, incorporel et intangible. Mais dans la réalité, souligne Commons, ces deux sortes sont souvent présentes ensemble dans un même actif, à la fois dette portant intérêt et revenus futurs anticipé. Toutefois, pour Commons, la caractéristique plénière du capitalisme contemporain est son inscription dans la temporalité de type flux-risque-profit, et dont le concept de capital intangible rend compte analytiquement.

Très tôt dans son œuvre, Commons a cherché à analyser le capital intangible. Dans son premier livre d'économie (Commons 1893), il caractérise ce capital à partir de l'idée selon laquelle les droits économiques sont des droits légaux qui permettent, à ceux qui les détiennent, le contrôle, garanti par la force publique, sur les actions des autres (1893, p. 62). Ces droits, le plus souvent d'ailleurs des faisceaux de droits (bundle of rights) (1893, p. 92), sont de différents genres, publics ou privés, partiels ou généraux. Commons décrit une variété de ces droits économiques (monopole, exclusivité, patente, franchise, copyright, etc.) et souligne que ceux-ci sont souvent des droits obtenus grâce à la souveraineté de l'État qui les accorde. Ces droits, qui résident souvent dans un rationnement de l'offre, offrent des positions de rente de situation (good-will) à ceux qui les détiennent. Souvent liés à des actifs, mais pas toujours, ils ne prennent pas nécessairement la forme juridique de titres légaux spécifiques bien qu'ils soient négociables. Ces droits, assis sur ces rentes de situation, constituent, pour Commons, un genre de capital qui n'a pas été identifié jusqu'à présent par les théories économiques ; il s'agit du capital intangible $e^{41}$.

Ce capital intangible n'est donc ni du capital corporel (du capital matériel) ni du capital incorporel (du capital immatériel mais tangible comme titre légal), il est la forme la plus évoluée du capitalisme moderne (1893, p. 89-91). Commons date la naissance légale de ce type de capital lorsque la Cour Suprême des États-Unis a, dans les années 1890, établi une définition d'une nouvelle sorte de propriété: la propriété intangible $\left(1933\right.$, p. 99 ${ }^{42}$. Commons résume cette définition comme «le droit de fixer les prix en refusant à autrui ce dont il a besoin mais qu'il ne possède pas » (1934a, p. 3) ; ou encore, "la propriété intangible est le droit de prendre des quantités espérées et de les évaluer à partir des ventes espérées de biens ou de services" (1934a, p. 522). Il s'agit donc bien d'un droit putatif sur les revenus qu'offre la futurité grâce à une rente de situation économique. Le capital intangible repose sur ce qui n'existe pas encore présentement mais dont la futurité est porteuse ; c'est un capital « futuristique » dit Commons. 

« un brevet, un copyright, une marque de commerce, une rente de situation, une réputation d'affaires, un bon crédit, un droit d'opérer une affaire, un droit d'accès à un marché du travail, un droit d'accès à un marché de biens ou à un marché monétaire, tout ce qui a une valeur présente basée sur des transactions anticipées d'achat ou de vente, s'empruntant ou se prêtant, s'embauchant ou se débauchant » (1924a, p. 159). De façon plus générale, une rente de situation est « l'anticipation quantifiable que des transactions économiques profitables pourraient être négociées, conclues et respectées dans l'avenir, en transigeant avec des personnes qui n'avaient auparavant pas de droits légaux ou éthiques pour acheter, vendre, ou fournir» $(1935$, p. 478). Cette rente de situation se concrétise pour le capital intangible qui la contrôle en « la détention présente de produits futurs ou de monnaie future dérivés de la vente de services et produits futurs » (1934a, p. 421). Pour Commons, le capital intangible exprime parfaitement la «futurité dans sa manifestation objective de la propriété» (1934a, p.418-419). Et ce processus d'objectivation de la futurité fait que le capital intangible « n'est pas un concept subjectif, il est la répétition anticipée de transactions profitables » (1933, p. 102).

Pour Commons, le concept de capital intangible permet de rendre compte plus pertinemment du processus capitaliste en ce qu'il est fondamentalement inscrit dans la futurité. Par exemple, en prenant une figure emblématique du capitalisme, «l'entrepreneur, lorsqu'il achète une marchandise n'achète pas un bien physique - il achète l'anticipation d'un revenu monétaire futur obtenu par la vente de la marchandise » (1934a, p. 429). De la sorte, «du point de vue de l'homme d'affaires, toute valeur marchande est un capital» (1924a, p. 159) et tout "actif est une propriété intangible incluant tout ce qui peut être vendu» (1924a, p. 158). Ainsi, le concept de capital intangible permet d'analyser « le véritable attribut du temps, attribut selon lequel les flux attendus de dépenses et de revenus déterminent, aussi bien en droit qu'en économie, la valeur actualisée des groupes actifs et des emplois des travailleurs » (1925, p. 378). un double sens. L'un est ce qui vient d'être présenté. Le capital intangible est totalement inscrit dans la futurité et non dans le présent ou le passé de l'accumulation. L'autre nouveauté, liée à ce premier aspect, est que le concept de capital intangible n'entretient plus de lien théorique avec le concept d'épargne. Ce capital n'est plus la figure inverse de l'épargne comme il l'est dans les théories économiques orthodoxes. L'épargne est classiquement attachée à un résultat du passé et est considérée comme la condition sine qua non du capital présent. Le capital intangible, lui, ne renvoie qu'à la futurité, sa valeur réside uniquement dans « l'anticipation escompté du revenu futur » (1934a, p. 455). Avec ces distinctions, on retrouve ce qui différencie les deux conceptions de la temporalité, celle de l'économie orthodoxe et celle de Commons. La première est celle d'un temps physique où l'épargne passée est la cause du capital présent. La seconde est une temporalité où la futurité, celle des anticipations putatives de revenus futurs, est la cause du capital intangible actuel. Plutôt que la temporalité de l'orthodoxie économique où temporalité et causalité ont le même sens physique, passé-présent-futur, la temporalité et la causalité économiques du capital intangible sont orientées dans le sens futuritéprésent. Ainsi, pour Commons, «la signification moderne du capital est entièrement séparée de la signification obsolète de l'épargne » (1934a, p. 456). À l'époque où règne le capitalisme bancaire, le capital, dans sa forme historique plénière, peut vivre et se 
développer sans « épargne préalable ». La perspective de profits permise par une futurité prometteuse, c'est-à-dire une rente de situation jugée profitable à terme, est suffisante pour que, sur cette base de revenus escomptés, un capital soit économiquement viable. La banque se fera un plaisir de créer une nouvelle quantité de monnaie de crédit en contrepartie d'une créance sur ce nouveau capital. Cette créance pourra alors entamer une vie active dans la futurité et peupler cette dernière d'une multitude de formes financières « dérivées » (réescompte, titrisation, etc.).

Le capital intangible est à la futurité ce que la dette est au passé. Dans une économie développée, l'un et l'autre sont tributaires de la monnaie. Dans le cas de la dette, la monnaie permet de quantifier et d'actualiser des actes économiques passés. Dans le cas $\mathrm{du}$ capital intangible, la monnaie permet de quantifier et d'actualiser des actes économiques inscrits dans la futurité. Ce sont les deux modalités par lesquelles la monnaie est active, sous forme d'actif capital incorporel-dette ou sous forme d'actif capital intangible-promesse ${ }^{43}$.

Avec ce concept de capital intangible, Commons met ainsi en évidence la logique profonde du capitalisme contemporain. Dès qu'une activité est, ou même simplement apparaît potentiellement, comme porteuse d'une perspective de revenus futurs importants, grâce à une rente de situation quelle qu'elle soit (technologie, exclusivité, monopole, brevet, copyright, etc.), elle est candidate à être du capital. Et ce capital intangible trouve essentiellement son expression concrète sous l'aspect d'un droit de propriété monétaire prenant la forme d'un actif financier. Ainsi analysée par Commons, la dynamique du capitalisme contemporain apparaît alors essentiellement comme soumise à une logique de financiarisation de l'activité économique. La logique du capitalisme n'est donc pas tant celle d'une «marchandisation» de toute chose, qu'une «capitalisation » de tout ce qui pourrait rapporter dans le futur, c'est-à-dire ce que la futurité promettrait en revenus à-venir. Ainsi compris, le capitalisme n'a plus de limite; toute activité est potentiellement « capitalisable ». La définition de l'« économie » prend alors une extension formidable. Un dirigeant d'entreprise, un joueur de football, un artiste, un savant, un concepteur de site informatique, un blogueur, etc. sont à l'origine de capitaux intangibles. Les jeunes pousses (startup) en font un mode de rémunération différée sous la forme d'options d'action (stock option). L'hégémonie capitaliste progresse ainsi avec la prolifération du capital intangible. Même les travailleurs ordinaires pourraient revendiquer de disposer d'un capital intangible. L'un des aspects du réformisme de Commons se comprend alors mieux. À l'exemple, notamment, de la négociabilité des dettes permise au dix-septième siècle anglais, de la définition de la propriété intangible au tournant du vingtième siècle états-unien (Commons 1924), Commons propose que le "fonds de commerce industriel des travailleurs" (1934a, p. 421), c'est-à-dire le «capital humain $»^{44}$ des travailleurs, dispose de la même sécurisation de leurs revenus futurs que les commerçants anglais du dix-septième siècle pour leurs effets de commerce, ou que les capitalistes états-uniens au tournant du vingtième siècle pour la sécurité de leurs investissements.

L'investissement productif ordinaire est aussi soumis à la logique du capital intangible. Une entreprise n'obtient un financement bancaire (crédit) ou financier (augmentation de capital) de ses investissements que si les valeurs anticipées de sa production et de ses profits, s'exprimant dans le cours des titres de propriété de cette entreprise (actions ou obligations) sont à un niveau suffisant, un certain multiple de la valeur de remplacement physique du capital productif (Commons 1934a, p. 601-602). Dans le capitalisme moderne, 
le capital intangible, c'est-à-dire le capital putatif existant dans la futurité et s'exprimant dans le cours des titres de propriété du capital, fonctionne donc comme le signal de la rentabilité du capital productif. Cette dernière est ainsi mesurée en termes de promesses de profit qu'offre une activité dans la futurité. Cette mesure de la rentabilité, qui s'exprime dans le capital intangible, fait disparaitre toute référence physique quant à la rentabilité du capital et son taux naturel d'intérêt. La productivité physique actuelle n'est pas le référent de la profitabilité du capital. Ce référent est dans la futurité, dans un (troisième $)^{45}$ monde qui n'est pas physique mais institué. Sous le capitalisme, une entreprise peut être physiquement productive sans pour autant être nécessairement profitable, tout dépend de sa situation dans la futurité, c'est-à-dire des anticipations qui sont faites sur les prix et revenus futurs qu'elle pourrait générer et que signale le cours des titres de propriété de cette entreprise. L'analyse en termes de détour de production ou en termes de taux marginal de productivité et de taux naturel d'intérêt, comme uniques références de profitabilité, perd alors une grande partie de sa pertinence. Commons propose alors de définir un taux de rentabilité du capital financier (capital-yield ) dont l'expression empirique serait un indice composé des principales valeurs de la bourse de New York (Commons 1934a, p. 603). Cet indice serait le référent comparatif des mesures de rentabilité pour les activités économiques. Ce référent, purement financier, serait en quelque sorte une évaluation quantitative de la futurité moyenne pour les transacteurs économiques.

Le concept de capital intangible met aussi en évidence l'une des spécificités historiques du capitalisme. Alors que les autres systèmes économiques passés ou étrangers, étaient, en général, essentiellement orientés vers la sécurisation de la futurité des (ordres de) transacteurs, pour leur avantage (citoyens libres, seigneurs, nobles, aristocrates, maîtres artisans, etc.) ou leur malheur (esclaves, serfs, domestiques, compagnons, apprentis, etc.) ; le capitalisme est un système économique en partie fondé sur la spéculation quant à la plus ou moins grande sécurité des transacteurs « libres » quant à leur futurité. Comme l'avait déjà remarquablement analysé T. Veblen (1904, Gislain 2000b), le capitalisme trouve sa survie et son dynamisme dans la vaste entreprise de sabotage économique menée par les capitalistes pour dégager des avantages différentiels, se concrétisant en profits. Les crises perpétuelles du capitalisme ne sont que les symptômes de sa raison d'être profonde et autant d'opportunités offertes à l'aventure capitaliste. De son côté, Commons montre remarquablement bien comment un certain degré d'ouverture plus ou moins sécuritaire de la futurité est la raison d'être du capital intangible. La futurité doit être suffisamment sécuritaire pour qu'une rente de situation soit capitalisable mais il faut aussi que cette situation ait un certain degré d'insécurité pour qu'elle apparaisse comme exceptionnellement rentable aux yeux des investisseurs. Une futurité totalement sécurisée n'offre pas de possibilité de capitalisation, elle offre seulement une rente au sens classique du terme. Une futurité totalement incertaine n'offre pas non plus de possibilité de capitalisation, aucun droit de propriété ne peut être protégé et conséquemment aucun revenu ne peut être raisonnablement anticipé ${ }^{46}$. La logique du capital intangible est de naviguer entre ses deux eaux troubles que sont la totale ou l'absence de sécurisation de la futurité des transacteurs ${ }^{47}$. Concernant la domination capitaliste, le thème de l'exploitation du travail a, à juste titre, beaucoup mobilisé la critique sociale du capitalisme, mais il serait aussi intéressant d'y ajouter le thème de l'insécurité de la futurité, notamment des travailleurs mais aussi de l'ensemble des transacteurs économiques. L'insécurité (ou la «liberté») quant à l'avenir constitue une part importante de la logique systémique du capitalisme. 
60 Avec son principe de futurité, et les développements analytiques qu'il permet et dont nous avons présenté quelques-uns ${ }^{48}$, Commons bouleverse la façon physicaliste dont l'économie orthodoxe appréhende la temporalité de l'activité économique. L'une des conséquences majeures de ce bouleversement est de faire apparaître un nouveau monde : le troisième monde des institutions économiques à l'origine des activités économiques.

61 Ce troisième monde s'articule aux deux autres mondes classiquement identifiés : celui du premier monde physique, objectif et extérieur, qui constitue l'environnement de l'activité humaine ; et celui du second monde singulier de la subjectivité personnelle et intérieure à chacun. Ces deux mondes sont régis par des temporalités spécifiques, physique pour le premier, psychologique pour le second. Les théories économiques orthodoxes appréhendent l'activité économique selon un rapport spécifique entre ces deux mondes. Dans le second monde, la psychologie est réduite à celle de la rationalité instrumentale et introspective de l'homo ceconomicus qui optimise son activité, en fonction de ses objectifs intérieurs au second monde et des contraintes du premier monde des ressources. L'activité économique y est donc comprise en analogie avec la physique et ses temporalité et causalité propres et identiquement orientées passé-présent-futur.

En introduisant le principe de futurité, tel que Commons nous le propose, l'activité économique peut être comprise du fait de l'existence d'un troisième monde, celui des institutions économiques. Celles-ci existent parce que, suivant la philosophie pragmatiste, le second monde comporte certes une capacité personnelle rationnelle étendue, celle d'expérimenter le monde et d'en tirer des apprentissages, ce que J. Dewey (1938) nomme l'intelligence, qui est essentiellement abductive; mais ce second monde a surtout reçu un bagage acquis et transmis collectivement concernant les modi operandi de l'activité, c'est-à-dire les institutions: les guides comportementaux de l'activité. Ces institutions ne sont donc pas à proprement parler personnelles (second monde), elles sont partagées et ont donc, de ce fait social (donc pas le premier monde), un sens objectif et une durée qui dépasse les individualités ${ }^{49}$ (donc pas le second monde). L'existence de ces institutions n'est pas essentiellement physique, même si elles peuvent se concrétiser matériellement, elle est surtout « dans les têtes » des transacteurs qui les font vivre en les adoptant, volontairement ou contraints, dans l'activité collective. Ces institutions sont " intangibles", et contrairement à une certaine tradition qui voudrait qu'elles s'inscrivent dans une durée issue du passé, elles se situent en fait dans la futurité, dans l'espace mental partagé de l'intentionnalité collective, dans une futurité significative commune ${ }^{50}$. Concrètement, pour Commons, « une institution sociale est un groupe actif ( going concern) ayant un projet, qui vit dans le futur mais agit dans le présent », et ceci, " car c'est la volonté humaine qui agit maintenant en vue de résultats futurs » (1934a, 619-620). L'univers institutionnel trouve son principe actif dans la futurité et les intentionnalités collectives qui s'y projettent. Grâce à cet univers, les acteurs économiques sont des esprits institués (institutionalized mind) qui agissent de concert ${ }^{51}$. Et, réciproquement, "seul un esprit institué peut élaborer le concept de temps » (1934a, p. 639) et dès lors se projeter dans la futurité.

63 Ainsi, la temporalité opérant dans ce troisième monde institutionnel a un sens causal inverse de la temporalité physique, elle s'oriente de la futurité vers le présent de l'activité. Pour Commons, c'est cette temporalité-là, celle à l'origine des institutions, qui est pertinente pour l'analyse de l'activité économique ; et dès lors, «la futurité est le principe le plus important de l'économie " (Commons 1934b, p. 125). Dans ces conditions, la domination économique s'établit certes sur un rapport exploiteur-exploité, mais aussi 
sur un rapport contrôle/non contrôle de la futurité. Dans la forme plénière du capitalisme et du capital intangible, les dominés sont exploités et/car privés du contrôle de leur avenir.

\section{BIBLIOGRAPHIE}

Adair P. (2013), « Cycle de crédit et politique monétaire : le monétarisme de John Rogers Commons ", Cahiers d'Économie Politique, n 64, p. 45-74.

Alary P. et al. (2016), Théories françaises de la monnaie, Paris, Puf.

Albert A. \& Y. Ramstad (1997), « The Social Psychology Underpinnings of Commons's Institutional Economics: The Significance of Dewey's Human Nature and Conduct », Journal of Economic issues, 31 (4), p. 881-916.

Albert A. \& Y. Ramstad (1998), « The Social Psychology Underpinnings of Commons's Institutional Economics II: The Concordance of George Herbert Mead's “Social Self” and John R. Commons's "Will" ", Journal of Economic issues, 32(1), p. 1-46.

Amable B. (2003), The Diversity of Modern Capitalism, Oxford, OUP.

Atkinson G. (2009), « Going Concerns, Futurity and Reasonable Value », Journal of Economic Issues, 43(2), p. 433-440.

Barber W.J. (2001), « From divergence to convergence. Irving Fisher and John R. Commons as champions of monetary reforms ", in J.E. Biddle et al. (ed.), Economic Broadly Considered, London, Routledge, p. 94-106.

Bazzoli L. (1999), L'économie politique de John R. Commons, Paris, L'Harmattan.

Becker G.S. (1964), Human Capital, Chicago, University of Chicago Press.

Becker H. (1963), Outsiders, Paris, A.M. Métailié, 1985.

Boyer R. \& Y. Saillard (2010), Théorie de la régulation, l'état des savoirs, Paris, La Découverte.

Broda P. (2015), « La place d'Industrial Goodwill dans la pensée de Commons », Cahiers d'Économie Politique, $\mathrm{n}^{\circ} 68$, p. 7-32.

Cassel G. (1903), The Nature and Necessity of Interest, London, Macmillan.

Clastres P. (1974), La Société contre l'État, Paris, Éditions de Minuit.

Commons J.R. (1893), The Distribution of Wealth, New York, Macmillan.

Commons J.R. (1995) [1924], Legal foundations of capitalism, New Brunswick, Transaction.

Commons J.R. (1925), « Law and Economics », Yale Law Journal, 34, February, p. 371-382.

Commons J.R. (1929a), Stabilization, Hearings before the Committee on Banking and Currency H.R. 11806, Washington, United States Government Printing Office, p. 56-104, p. 423-444.

Commons J.R. (1929b), « The definition of price », Research in the History of Economic Thought and Methodology, vol. 18, p. 309-334. 
Commons J.R. (1933), « Materialistic, Psychological, Institutional Economics », Economic Essays in Honour of Gustac Cassel, London, Allen \& Unwin, p. 89-103.

Commons J.R. (1990) [1934a], Institutional Economics. Its Place in Political Economy, New Brunswick, Transaction.

Commons J.R. (1934b), Myself, New York, Macmillan

Commons J.R. (1935), « The Place of Economics in Social Philosophy ", Journal of Social Philosophy, I, October, p. 7-22; repris dans (1996, II), p. 475-486.

Commons J.R. (1939), Investigational Economics, edited by Warren J. Samuels in Research in Economic Thought and Methodology, Archival supplement 7, 1998, p. 39-326.

Commons J.R. (1996), John R. Commons: Selected Essays [M. Rutherford \& W.J. Samuels (eds)], London, Routledge, 2 vol. I et II.

Costa Isabel (da) (2010), « L'institutionnalisme de John Commons et les origines de l'État providence aux États-Unis », Interventions économiques, $\mathrm{n}^{\circ} 42$.

Darwin C. (1999) [1859], L'origine des espèces, Paris, Flammarion.

Derrida J. (1967), La Voix et le Phénomène, Paris, Puf.

Descombes V. (1996), Les institutions du sens, Paris, Éditions de Minuit.

Dewey J. (2003) [1927], Le public et ses problèmes, Pau, Farrago-Léo Scheer.

Dewey J. (1993) [1938], Logique. La théorie de l'enquête, Paris, Puf.

Dewey J. (1972-1985), The Early Works, 1882-1898; The Middle Works, 1899-1924 ; The Later Works, 1925-1953 [J.A. Boydston (ed.)], Carbondale, Southern Illinois UP, 37 vol.

Dubar C. (2014), « Du temps aux temporalités : pour une conceptualisation multidisciplinaire », Temporalités [En ligne], 20 | 2014, mis en ligne le 24 février 2015, consulté le 2 janvier 2018.

URL :http://journals.openedition.org/temporalites/2942 ;

DOI : $10.4000 /$ temporalites.2942

Dubar C. \& J. Thoemmes (dir.) (2013), Les temporalités dans les sciences sociales, Toulouse, Octares.

Durkheim E. (1894), Les règles de la méthode sociologique, Paris, Alcan.

Durkheim E. (1975) [1900], « La sociologie et son domaine scientifique », in Texte 1 : Éléments d'une théorie sociale, Paris, Éditions de Minuit, p. 13-36.

Elias N. (1996) [1984], Du temps, Paris, Fayard.

Fisher I. (1906), The Nature of Capital and Income, London, Macmillan.

Fisher I. (1932), Booms and Depressions, New York, Adelphi.

Gislain J.-J. (1999), « Les conceptions évolutionnaires de T. Veblen et J.R. Commons », Économies et Sociétés, HS n³4, p. 47-65

Gislain J.-J. (2000a) « L'artifice économique chez T. Veblen et J.R. Commons : surplus et rareté, pouvoir et ordre ", Cahiers du GRATICE, n 19 : «L'institutionnalisme, histoire et analyse ", p. 37-57.

Gislain J.-J. (2000b) « La naissance de l'institutionnalisme : Thorstein Veblen », in A. Béraud \& G. Faccarello (dir.), Nouvelle histoire de la pensée économique, Paris, La Découverte, Tome III, Chapitre XXX, p. 74-115. 
Gislain J.-J. (2002), « Causalité institutionnelle : la futurité chez J.-R. Commons », Économie \& Institutions, 1(1), p. 47-66.

Gislain J.-J. (2003a), « L'institution des relations industrielles : le cadre analytique de J.R. Commons ", Économie \& Institutions, 1(2), p. 9-56.

Gislain J.-J. (2003b), « L'émergence de la problématique des institutions en économie », Cahiers d'Économie Politique, n 44, p. 19-50.

Gislain J.-J. (2004), « Futurité et toposité : situlogie des perspectives de l'action », Géographie, Économie, Société, 6(2), p. 203-219.

Gislain J.-J. (2006), « Le processus d'innovation sociale : un cadre d'analyse institutionnaliste pragmatiste ", in P.A. Lapointe \& G. Bellemare (dir.), Innovations dans le travail et l'emploi. Recherches empiriques et perspectives théoriques, Québec, Presses de l'Université Laval, p. 237-274. Gislain J.-J. (2011a) « Pourquoi l'économie est-elle nécessairement instituée ? Une réponse commonsienne à partir du concept de futurité », Interventions Économiques, nº 42, 19 p.

Gislain J.-J. (2011b), « Le darwinisme méthodologique de Veblen », Économie \& Institutions, $\mathrm{n}^{\circ} 17$, p. 7-23.

Gislain J.-J. (2017), « Futurité, la temporalité économique chez J.R. Commons », Economia, 7(2), p. 239-270.

Graeber D. (2013) [2011], Dette : 5000 ans d'histoire, Paris, Les liens qui libèrent.

Guerrien B. \& E. Benicourt (2008), La théorie économique néoclassique, Paris, La Découverte.

Hall E.T. (1984) [1983], La Danse de la vie. Temps culturel, temps vécu, Paris, Seuil.

Hall P.A. \& D. Soskice (eds) (2001), Varieties of Capitalism, Oxford, OUP.

Hawtrey R.G. (1935) [1919], La circulation monétaire et le crédit, Paris, Recueil Sirey.

Heidegger M. (1986) [1927], Être et le temps, Paris, Gallimard.

Hodgson G.M. (2004), The Evolution of Institutional Economics: Agency, Structure and Darwinism in American Institutionalism, London, Routledge.

Keen S. (2014) [2011], L'imposture économique, Paris, Atelier.

Keynes J.M. (1930), A Treatise on Money, London, Macmillan, 2 vol.

Kirat T. \& L. Vidal (dir.) (2011), Le capitalisme au futur antérieur, Bruxelles, Bruylant.

Klein É. (2009), Les tactiques de Chronos, Paris, Flammarion.

Knapp G.F. (1924) [1905], The State Theory of Money, London, Macmillan.

Leccardi C. (2014), « Time of society and time experience: multiple times and social time », Kronoscope, $\mathrm{n}^{\circ} 14$, p. 10-24.

MacLeod H.D. (1923) [1856], The Theory and Practice of Banking, London, Longmans, 2 vol.

MacLeod H.D. (1867), The Elements of Economics, London, Longmans, 1881, 2 vol.

Maucourant J. (1993), « Au cœur de l'économie politique, la dette - l'approche de J.R. Commons », Revue du MAUSS, $2^{\mathrm{e}}$ sem., p. 209-218.

Maucourant J. (2001), «L'institutionnalisme de Commons et la monnaie », Cahiers d'économie politique, vol. 40-41, p. 253-284.

Mead G.H. (2012) [1932], La philosophie du temps en perspective(s), Paris, éditions de l'EHESS. 
Mead G.H. (2006) [1934], L'esprit, le soi et la société, Paris, Puf.

Mirowski P. (1987), « The philosophical bases of institutionalist economics », in M. R. Tool (1988) (ed.), Evolutionary economics, Armonk, Sharpse, vol. I, p. 51-88.

Moran C. (2015), « Time as a social practice », Time \& Society, 24(3), p. 283-303.

Morel S. (2000), Les logiques de la réciprocité : les transformations de la relation d'assistance aux ÉtatsUnis et en France, Paris, PUF.

North D. (1990), Institutions, Institutional Change and Economic Performance, Cambridge, CUP.

Olson M. (1978) [1966], Logique de l'action collective, Paris, Puf.

Orlean A. (2005), « Réflexions sur l'hypothèse d'objectivité de la valeur fondamentale dans la théorie financière moderne ", in D. Bourghelle et al. (dir.), Croyances, représentations collectives et conventions en finance, Paris, Economica, p. 19-42.

Ostrom E. (2010) [1990], Gouvernance des biens communs, Louvain-la-Neuve, De Boeck.

Palan R. (2015), « Futurity, Pro-cyclicality and Financial Crises », New Political Economy, 20(3), p. 367-385.

Parsons T. (1951), The Social System, London, Routledge \& Kegan Paul.

Peirce C.S. (1984) [1878], « Comment rendre nos idées claires? », dans Textes anticartésiens, Paris, Aubier, p. 287-308.

Peirce C.S. (1931-1958), Collected Papers, Cambridge (Mass.), Harvard UP, 8 vol.

Ramstad Y. (1986), « A pragmatist's quest for holistic knowledge: the scientific methodology of John R. Commons », repris dans W. Samuels (1988) (ed.), Institutional Economics, Aldershot, E. Elgar, vol. II, p. 138-176.

Renault M. (1999), «Économie et coordination des comportements : communication et interaction ", Revue Européenne des Sciences Sociales, t. 37, n 114, p. 265-292.

Renault M. (2000), «Évolutionnisme et pragmatisme : Veblen, Dewey, Commons, Fondements philosophiques », Cahiers du GRATICE, n 19, p. 181-206.

Renault M. (2009), « Perspectivisme, moralité et communication. Une approche transactionnelle de la responsabilité sociale des entreprises », Revue française de socio-économie, $\mathrm{n}^{\circ}$ 4, p. 15-37.

Revue Économique (1989), L'économie de convention, $\mathrm{n}^{\circ} 2$, vol. 40.

Schnell A. (dir.) (2007), Le temps, Paris, Vrin.

Searle J.R. (1998) [1995], La construction sociale de la réalité, Paris, Gallimard.

Simmel G. (1987) [1900], Philosophie de l'argent, Paris, Puf.

Sumner W.G. (1906), Folways, a study of the sociological importance of usages, manners, customs, mores, and morals, Boston, Ginn and Co.

Spencer H. (1862), First Principles, London, Williams and Norgate.

Testart A. (dir.), Aux origines de la monnaie, Paris, éditions errance.

Tool M.R. (1994), « An institutionalist mode of inquiry: limitations of orthodoxy », in P.A. Klein (ed.), The Role of Economic Theory, Dordrecht, Kluwer, p. 197-227.

Veblen T. (1970) [1899], Théorie de la classe de loisir, Paris, Gallimard.

Veblen T. (1927) [1904], The theory of business enterprise, New York, Ch. Scribner's sons. 
Veblen T. (1965) [1921], The engineers and the price system, New York, M. Kelley.

Weber M. (1971) [1921], Économie et Société, Paris, Plon.

Wicksell K. (1898), Geldzins und Güterpreise, Jena, G. Fischer.

Zawadzki P. (dir.) (2002), Malaise dans la temporalité, Paris, Publications de la Sorbonne.

\section{NOTES}

1. Sur l'émergence de la problématique des institutions en économie, voir J.-J. Gislain (2003b).

2. Pour une présentation générale de l'approche de J.R. Commons, voir l'ouvrage de L. Bazzoli (1999). Pour une comparaison des approches de Veblen et Commons, voir J.-J. Gislain (2000a).

3. Ces principes sont des « hypothèses logiques, faites dans le but d'attendre une interprétation et une compréhension systématiques de la diversité du monde. Ce sont des instruments pour la recherche. La validité de ces hypothèses réside dans leur usage fructueux " (Commons 1939, p. 97-98).

4. Trois revues sont spécialisées dans ce domaine : Time \& Society, Temporalités et Kronoscope. Pour une synthèse sur la pluralité des temporalités voir les articles de C. Laccardi (2014), C. Moran (2015), C. Dubar (2014), et les ouvrages collectifs de P.Zawadzki (dir.) (2002) et C. Dubar. \& J. Thoemmes (dir.) (2013). D'un point de vue plus anthropologique, voir aussi la typologie en forme de mandala des différents temps de E.T. Hall (1983, p. 238).

5. «[...] le principe de futurité est séparable en pensée mais inséparable dans les faits des principes de rareté et d'efficacité » (Commons 1934a, p. 389).

6. Concernant l'influence de la philosophie pragmatiste sur Commons, voir Y. Ramstad (1986), P. Mirowski (1987), A. Albert \& Y. Ramstad $(1997,1998)$ et M. Renault (2000).

7. Commons définit le groupe actif (going concern) comme « une action concertée existant tant que le groupe est actif et anticipe de futures transactions profitables. Quand la futurité s'arrête le groupe arrête d'aller» (1933, p. 99-100).

8. La « coutume qui est une sorte de contrainte imposée aux individus par l'opinion collective sur ce que l'on ressent et fait pareillement » (1934a, p. 153).

9. Dans la hiérarchie catégorielle, Commons classe le principe au-dessus du concept et lui accorde donc un ordre de généralité plus important.

10. Commons appréhende l'acteur économique en tant que "transacteur », c'est-à-dire un acteur qui est toujours en position réciproque avec au moins un autre acteur dans une «trans-action ». Pour analyser l'activité économique, Commons propose une typologie des transactions en trois types régis respectivement par un principe: les transactions de marchandage (rareté), de direction (efficacité) et de répartition (souveraineté). De plus, pour Commons, appréhender l'économie d'un point de vue transactionnel implique nécessairement d'intégrer dans la théorie économique la dimension du temps alors que «le concept de temps pouvait ne pas avoir d'importance tant que la théorie économique en restait aux marchandises, sans passer aux transactions» (1934a, p. 84).

11. Une coutume peut aussi être le fondement d'une politique publique. Pour une étude complète sur cette question voir S. Morel (2000).

12. Sur la conception générale de l'évolution des institutions chez Commons, voir J.-J. Gislain (1999). Pour une application de cette conception au cas de l'innovation sociale, voir J.-J. Gislain (2006).

13. Sur l'évolution de la doctrine réformiste de Commons, voir P. Broda (2015).

14. Voir infra. 
15. Pour une application de cette même conception pragmatiste mais cette fois à la dimension spatiale de l'activité, voir J.-J. Gislain (2004).

16. Pour Commons, le transacteur est « un corps entier en action [...], un acteur créatif orienté vers le futur, manipulant le monde extérieur et les autres en vue de conséquences anticipées » (1934a. p. 17).

17. Voir sur ce point N. Elias (1984).

18. Sur les débats actuels concernant la notion de temps en science physique, voir É. Klein (2009) et sur les différentes philosophies du temps voir A. Schnell (dir.) (2007).

19. À cet égard, est étonnante la tradition intellectuelle qui consiste à relier-comparer-etc. le passé, le présent et le futur, alors que ce dernier est radicalement différent, n'existant pas, des deux premiers qui eux existent bien. Il est donc plus logique de mettre en relation ce qui est comparable, le passé, le présent et la futurité, plutôt que d'introduire un élément de comparaison hétérogène, le « futur ».

20. On pourrait rapprocher cette approche de Commons de celle du «perspectivisme» de G.H. Mead (1932). Voir à cet égard A. Albert \& Y. Ramstad (1998) et M. Renault $(1999,2007)$.

21. C'est une des similitudes importantes entre les approches de la temporalité chez Commons et J.M. Keynes qui discrédite la théorie néoclassique des anticipations rationnelles. Pour Commons, «Ce n'est pas un état rationnel de la société qui détermine l'action, c'est un ensemble merveilleusement irrationnel et complexe d'anticipations auquel sont confrontés les participants aux transactions » (Commons 1934a, p. 683).

22. Dès 1925, Commons défend la conception selon laquelle la «futurité est commune aux sciences économique et juridique et les distingue des sciences physiques. Quand les économistes ont fondé leurs théories sur des analogies avec les sciences physiques, desquelles le principe d'efficacité est dérivé, ils représentaient la conduite humaine comme une résultante de forces physiques qui poussaient l'homme du passé dans le présent. L'individu était l'objet de lois "naturelles" auxquelles il ne pouvait pas déroger. La soi-disant "loi de l'offre et de la demande", dérivée du principe de rareté, était une force physique de cette sorte. Et en accord avec ce qui précède, le concept de temps, dans les sciences physiques, est celui d'un flux d'événements procédant inévitablement d'un antécédent vers un conséquent. Mais lorsque nous regardons la volonté humaine (human will), ou plutôt le vouloir humain (human willingness), comme la force avec laquelle la science économique a à faire, en contraste avec les forces physiques de la gravitation, de l'électricité, de la mécanique, et ainsi de suite, alors nous trouvons que ce sont les espoirs et les craintes, les attentes et les précautions, la prévoyance et l'impatience, inscrites dans le futur qui déterminent ce qui sera fait dans le présent. Nous avons, en économie et en droit, plusieurs termes indiquant cet aspect futuriste du temps comme motif, intention, projet, besoin, désir, sécurité, investissement, propriété, actifs, dettes, intérêt, capital - en fait, le concept de valeur lui-même, autour duquel tourne la théorie économique, aussi bien que la théorie juridique, est une synthèse de tous ces autres concepts de futurité et, comme tel, est toujours un concept de l'importance présente des choses, des personnes et des classes de personnes en vue de leur usage et comportement attendus dans un futur immédiat ou éloigné. C'est en vérité, ce principe de futurité qui change le concept usuel de la nature humaine développé par les économistes" (1925a, p. 377). Voir aussi sur ce point G. Atkinson (2009, p. 435-437).

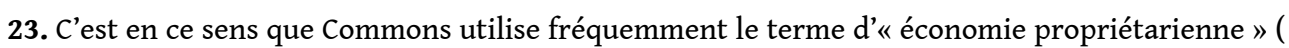
proprietary economics) comme synonyme d'économie institutionnaliste (institutional economics).

24. On peut dire que les institutions, du fait qu'elles structurent la futurité des transacteurs, sont performatives : elles « ordonnent » (disent et organisent) les perspectives d'activité.

25. Pour une application de ce concept au domaine des relations industrielles, voir J.-J. Gislain (2003a). 
26. Dans la tradition pragmatiste le concept d'«action collective» ne signifie pas l'action d'un « collectif » d'individus en tant qu'entité sociale agissant comme un «individu collectif » (Olson 1966), il doit être compris comme l'ensemble des (trans-)actions individuelles par/dans un collectif. H. Becker en donne une définition très claire: L'action collective est «l'ajustement réciproque des lignes d'action ", c'est-à-dire que les individus « font ce qu'ils font avec un œil sur ce que les autres ont fait, sont en train de faire, ou sont susceptibles de faire dans le futur. Les individus cherchent à ajuster mutuellement leurs lignes d'actions sur les actions des autres perçues ou attendues. On peut appeler action collective le résultat de tous ces ajustements, surtout si l'on garde présent à l'esprit que le terme ne renvoie pas aux seules actions collectives explicitement concertées, mais recouvre toutes les activités que l'on peut considérer comme accomplies par un certain nombre de gens agissant ensemble " (Becker 1963, 205-206). Commons (1934a) différencie les actions collectives en inorganisées ou organisées selon qu'elles sont régies par des règles opérantes de conduite (working rules) informelles ou formelles. Commons avait initialement proposé le concept d'" action concerté » (1931) mais ensuite il a repris le concept plus classique d'«action collective ». Il serait aussi possible de donner à ce concept le nom d'« activité collective ».

27. Pour Commons (1934a, p. 73) les institutions « contraignent, libèrent ou étendent » l'activité des transacteurs.

28. «Faire l'impasse sur la monnaie en la considérant comme superficielle a rendu l'économie orthodoxe incapable de traiter de l'économie moderne » (Commons 1934a, p. 190).

29. «La monnaie n'a pas de valeur d'usage, elle n'est que la "forme" de la valeur dans le processus physique de l'échange » (1934a, 372).

30. «La monnaie n'est qu'une valeur nominale » (1934a, p. 130). «Sous cet angle, la valeur-rareté peut être transposée de l'économie psychologique vers l'économie institutionnelle, où les propriétés sont aliénées et acquises grâce aux transactions. Ici, l'unité de mesure de la rareté est une autre institution, la monnaie, et le nom approprié est le prix, au lieu du terme psychologique d'“utilité marginale"» (1934a, p. 85-86).

31. "Lorsque j'achète un cheval ou une terre, ce que j'achète n'est pas la chose physique, c'est l'ensemble des droits, "opposables au monde entier", sur les futures utilisations du cheval ou de la terre » (Commons 1934a, p. 416).

32. "Nous mesurons une variation de chaleur d'une façon indirecte en mesurant ses effets sur une colonne de mercure arbitrairement graduée en unités de longueur définies. De la même façon, nous mesurons les variations de la rareté par leurs effets sur le prix payé pour une unité de marchandise rare exprimée arbitrairement en unités de dollars et de cents » (1934a, p. 262).

33. "Sa variabilité (de l'unité monétaire) doit être corrigée à un niveau convenu de pouvoir d'achat moyen à un moment particulier, par exemple l'année 1913 ou l'année 1926, et ses variations de valeur-rareté sont alors l'inverse de la variation au-dessus ou au-dessous du niveau moyen du pouvoir d'achat. À partir de ce niveau de référence, les variations des raretés relatives pour des marchandises données deviennent la "dispersion» de leurs prix par rapport à la moyenne. Ainsi donc le pouvoir d'achat moyen est-il un palliatif statistique à la difficulté de distinguer la valeur nominale de la valeur réelle. Ce n'est qu'une unité de mesure de la valeurrareté de la monnaie, qui varie en fonction inverse de son pouvoir d'achat moyen. Lorsque les prix augmentent, la valeur de l'unité monétaire baisse et lorsque les prix baissent, la valeur de l'unité monétaire augmente. C'est cette moyenne, et non pas une notion de valeur réelle, qui sert de référence à partir de laquelle mesurer la dispersion de prix particuliers. C'est une théorie de la mesure et non une théorie de la valeur, réelle ou nominale. Ainsi le statisticien, prenant le pouvoir d'achat moyen de la monnaie en 1913 comme base 100, mesure-t-il, par leur écart par rapport à la moyenne, les variations ultérieures des raretés relatives des marchandises particulières, quelles que soient les causes de ces variations. Au cours d'une période ultérieure, la moyenne des prix des mêmes marchandises pourra avoir augmenté de $10 \%$, faisant apparaître 
une diminution de $9 \%$ de la rareté de la monnaie relativement à la moyenne de toutes les autres raretés » (1934a, p. 262).

34. «Dans les temps anciens, la monnaie était une simple monnaie de compte, comme le bœuf en Grèce ; elle devint ensuite une marchandise métallique. Puis les rois estampèrent le métal et en firent le moyen légal de paiement de l'impôt et des dettes privées. Les pièces de monnaie cessèrent alors d'être des marchandises. Elles devinrent une institution, à savoir le cours légal, moyen collectif permettant de s'acquitter des dettes publiques et privées » (1934a, p. 392).

35. Il ne peut d'ailleurs en être autrement car, pour Commons, "c'est le lien de l'argent qui fait tenir ensemble la "société"; qu'il vienne à casser et la société tombe en morceaux » (1934a, p. 123).

36. Sur sa théorie des prix, voir Commons (1919b).

37. Pour Commons, "la monnaie est secondairement un medium de l'échange, elle est premièrement un moyen de créer et de payer des dettes » (1929b, p. 326). Sur cette question, voir aussi J. Maucourant $(1993,2001)$. Par ailleurs, on ne peut s'empêcher de trouver de nombreuses similitudes entre Commons et, actuellement, les «théories françaises de la monnaie » (Alary et al. 2016).

38. Pour Commons (1934a, 512), conformément à sa conception endogène de la monnaie, dans l'équation $\mathrm{MV}=\mathrm{PT}$, c'est $\mathrm{P}$ (le niveau des prix) qui est la cause de $\mathrm{M}$ (masse monétaire) et non l'inverse (V étant la vitesse de circulation de la monnaie et $\mathrm{T}$ la quantité de transactions) comme traditionnellement dans la théorie quantitative de la monnaie.

39. Sur cette question, voir et W.J. Barber (2001) et P. Adair (2013).

40. Commons reconnaît à G. Cassel (1903) d'avoir théoriquement distingué «la propension à attendre et la propension à courir des risques » (Commons 1934a, 503).

41. T. Veblen (1904) a aussi élaboré le concept de capital intangible mais pour Commons Veblen n'a pas vu que l'« intangibilité réside non dans la simple immatérialité mais dans le fait d'être affaire de futurité » $(1950,106)$.

42. Commons a analysé en détail l'apparition et l'évolution historiques de ce capital intangible dans ses Legal Foundations of Capitalism (1924).

43. En termes hippiques, le mot «futurité » sert à nommer un « espoir », c'est-à-dire un jeune cheval qui promet pour l'avenir.

44. Ce concept élaboré plus tard par G. Becker (1964), dans le cadre de la théorie néoclassique, est une version très différente de celle de Commons, bien que son origine commune se trouve dans la tradition néo-autrichienne états-unienne du début du vingtième siècle, notamment chez I. Fischer (1906)... dont Commons, sur certains aspects analytiques, est assez proche.

45. Voir plus bas.

46. L'approche néo-institutionnaliste en histoire développe cette problématique.

47. Pour une analyse de l'instabilité cyclique du capitalisme financiarisé du fait de cette dualité de la futurité, voir R. Palan (2015). Pour une analyse de cette dualité à l'œuvre au cours de l'évolution des institutions juridiques dans le capitalisme français des deux derniers siècles, voir T. Kirat \& L. Vidal (2011).

48. Pour d'autres développements, voir J.-J. Gislain (2017).

49. La sociologie durkheimienne aborde aussi cette question mais sans mettre en évidence la temporalité propre des institutions et son principe de futurité. En philosophie, les approches de J.R. Searle (1995) et V. Descombes (1996) analysent bien cette idée d'objectivité des institutions. En théorie économique, A. Orléan exprime bien ce qui différencie l'approche orthodoxe de celle proposée ici : «On passe d'un monde où est postulée l'existence d'une vraie représentation du futur, donnée objectivement, à un monde dans lequel la représentation du futur n'est pas un fait de nature, mais est le résultat d'un processus collectif de croyance partagée. Elle n'est pas naturelle mais historiquement et socialement construite. » (Orléan 2005, p. 20) 
50. «La vision institutionnelle est plutôt intangible parce que toutes les choses physiques qu'elle prend en considération sont situées dans un futur proche ou lointain et n'existent qu'à travers les anticipations actuelles sur la permanence de l'action collective " (Commons 1934a, p. 618).

51. "L'homme est davantage qu'un organisme, il est institutionnisme [institutionism] et il n'y a que l'esprit institutionnalisé (institutionalized mind) pour développer cette remarquable dimension temporelle de l'activité économique que nous avons appelée la futurité. La futurité est institutionnelle ; tout comme l'animal, l'enfant en bas âge et l'homme abandonnés à eux-mêmes, ne sauraient à peu près rien, voire rien du tout, à son sujet. Cette extension institutionnelle du cerveau des organismes vers le futur éloigné est inséparable de son extension dans l'espace éloigné. Ce sont ces deux extensions institutionnalisées de l'activité du cerveau qui rendent possibles les groupes actifs [going concerns] modernes très développés au sein de l'industrie et de l'État qui donnent des ordres dans le monde entier et aux générations à venir » (Commons 1934a, p. 639).

\section{RÉSUMÉS}

L'approche institutionnaliste de J.R. Commons est la plus apte à expliquer l'origine des institutions économiques. Elle met en évidence le rôle crucial qu'y joue le principe de futurité. Ce dernier imprime un genre de causalité spécifique, la causalité institutionnelle, à l'œuvre dans l'activité économique. Les institutions centrales du capitalisme moderne, la monnaie de crédit et le capital intangible, sont l'expression exemplaire du principe de futurité.

The institutionnaliste approach of J.R. Commons is the most capable of explaining the origin of the economic institutions. It highlights the crucial role which plays the principle of futurity. This principle prints a kind of specific causality, the institutional causality, in the work in the economic activity. The central institutions of the modern capitalism, the currency credit and the intangible capital, are the exemplary expressions of the principle of futurity.

\section{INDEX}

Keywords : J.R. Commons, futurity, institution, institutional causality, currency credit, intangible capital

Mots-clés : J.R. Commons, futurité, institution, causalité institutionnelle, monnaie de crédit, capital intangible

\section{AUTEUR}

\section{JEAN-JACQUES GISLAIN}

Centre de recherche sur les innovations sociales (CRISES) (Québec, Canada) ; jeanjacques.gislain@rlt.ulaval.ca. 